\title{
Introduction to special issue on 'Advances in Information Systems and Network Systems Security'
}

\author{
Sanjay K. Jena ${ }^{1}$ - Ashok K. Turuk ${ }^{1} \cdot$ Manmath N. Sahoo $^{1} \cdot$ Sambit Bakshi $^{2}$
}

Published online: 21 June 2015

(c) Springer-Verlag London 2015

2nd International Conference on Advanced Computing, Networking and Informatics (ICACNI-2014), held on 24-26 June 2014, was organized by St. Thomas' College of Engineering and Technology. The conference was technically co-sponsored by IEEE Computer and IEEE Computational Intelligence Societies, Kolkata Chapter. It was inaugurated on 24th June, 2014 in the famous Parish Hall, located inside the holy place of St. Paul's Cathedral, Kolkata. The conference received 646 technical submissions, of which only 148 papers were accepted based on at least two single-blind reviews. Along with presentations in three main tracks: Advanced Computing, Networking, and Informatics, ICACNI hosted three special sessions for discussion of specific interest areas. ICACNI included 20 technical sessions spread over 3 days during 24-26 June, 2014. There were four parallel sessions with a total of 148 paper presentation slots. The Proceedings of the Conference has been published in two volumes in the prestigious Smart Innovation Systems and Technologies Series, Springer. The conference, in its second edition, not only explored and recorded cutting-

Sambit Bakshi

sambitbaksi@gmail.com

Sanjay K. Jena

skjena@nitrkl.ac.in

Ashok K. Turuk

akturuk@nitrkl.ac.in

Manmath N. Sahoo

sahoom@nitrkl.ac.in

1 Department of Computer Science and Engineering,

National Institute of Technology Rourkela,

Rourkela 769 008, Odisha, India

2 Department of Computer Science and Engineering, National Institute of Technology Jamshedpur, Jamshedpur 831 014, Jharkhand, India edge advancements in the field of Computer Science, but also brought into light several technical challenges that would be the research objectives for next decade.

Among 148 articles accepted in ICACNI, few high-quality articles were invited to submit to this special issue of Innovations in Systems and Software Engineering. Finally, after careful review by reputed academicians, only five articles are accepted to be published in the special issue. The articles span from privacy preservation, new authentication models to analysis of rhythm in music. The following briefs the articles available in the issue.

The first article titled "On Analysis of Time Series Data with Preserved Privacy" authored by Sarat Kumar Chettri and Bhogeswar Borah addresses the issue of analyzing numerical time-series of equal length with preserved privacy. The discrete wavelet transform-based proposed method is experimentally established to be superior to the existing methods in preserving the trade-off between data utility and privacy.

The second article titled "On Vulnerability Analysis of Several Password Authentication Protocols" authored by Keith Garrett, S. Raghu Talluri, and Swapnoneel Roy formulates a generalized algorithm for cryptanalysis to perform clogging attack (a form of denial of service) on protocols which use the computationally intensive modular exponentiation to guarantee security. This proposed technique is applied to cryptanalyse some recent password authentication protocols.

The third article titled "Spanning Tree-Based Fast Community Detection Methods in Social Networks" authored by Partha Basuchowdhuri, Riya Roy, Siddhartha Anand, Diksha Roy Srivastava, Subhashis Majumder, and Sanjoy Kumar Saha presents two maximum cost spanning tree-based community (defined as dense subgraphs present in social networks) detection methods, namely P-SPAT and K-SPAT, for social networks. Detecting communities in a social network 
is a challenging task, in terms of computational overheads and accuracy of the detected communities. The proposed work is tested on benchmark datasets and is claimed to be able to detect communities with high accuracy.

The fourth article titled "Rhythm Analysis of Tabla Signal by Detecting the Cyclic Pattern" authored by Susmita Bhaduri, Orchisama Das, Sanjoy Kumar Saha, and Chandan Mazumdar presents mechanism to detect two important rhythmic aspects of tala namely, tempo and matra. It is focused on the detection of the repeating structure by analyzing the tabla signal. The work is tested on a wide variety of electronic tabla signals (the repetition is perfectly cyclic) as well as signal captured when tabla is played by human artist (the cycle duration may vary).

The fifth and final article of this special issue is titled "Region Based Feature Extraction from Non-Cooperative Iris Images Using CDF 9/7 Filter Bank" authored by Soub- hagya Sankar Barpanda, Banshidhar Majhi, and Pankaj K. Sa. The article deals with application of CDF 9/7 filter bank for extracting region-based feature from iris images to establish uniqueness. The feature thus extracted being a region-based feature bears the combinational quality of both local and global features. The technique may find its application in any biometric system established in any organization.

We are sure that readers would find the content of the issue scientifically very significant. We would like to express our gratitude to the reviewers for their insightful comments to improve the quality of submitted articles and discarding articles with less significance. We would also like to express our deepest thanks to all the authors who considered submitting articles for this special issue. We would conclude with acknowledging the Editor-in-Chief, Prof. Michael G. Hinchey, for his support to publish this special issue and constant encouragement to maintain fine quality. 